\title{
Softening of Silica Filled Styrene-Butadiene Rubber under Uniaxial Cyclic Loading
}

\author{
Olesja STARKOVA*, Andrey ANISKEVICH
}

\author{
Institute of Polymer Mechanics, University of Latvia, Aizkraukles 23, LV-1006, Riga, Latvia \\ cross ${ }^{\text {ref }}$ http://dx.doi.org/10.5755/j01.ms.22.2.7953
}

Received 08 September 2014; accepted 25 January 2015

\begin{abstract}
Stress-strain behaviour of silica filled styrene-butadiene rubber showed strong hysteresis and cyclic softening, while these effects were almost negligible for the neat rubber. Significant Mullins effect occurs between the first and second loading cycles, whereupon samples reach their steady state after four repeated cycles. The degree of softening increases with volume content of silica characterizing the softening effect as a breakdown of the filler-formed microstructure defined by both, specific interactions in the filler associations and between the filler and rubber matrix. Contribution of different damage mechanisms to the total energy losses on different stages of extension was estimated by strain dependences of the hysteresis losses for different loading cycles. At the very beginning of deformation, energy losses of the filled rubber are associated with intensive destruction of the filler associations, while under large elongations the main contribution comes from the breakage of bonds between the rubber chains and fillers and from the rubber internal viscosity. After repeated loading-unloading cycles, the material structure is practically recovered in each cycle and hysteresis losses are caused by changes in the rubbery phase only. Ability of the neat and filled rubber to almost completely restore their structure is confirmed by reversible volume changes.

Keywords: filled rubber, Mullins effect, stress softening, hysteresis losses.
\end{abstract}

\section{INTRODUCTION}

Rubbers and rubber-like materials are used in a wide variety of different industrial and automotive areas. The mechanical properties, e.g. elastic modulus and tensile strength, of such materials can be substantially improved by incorporating small particle-size fillers to a crosslinked elastomer matrix [1-6]. The most widely used filler for rubbers, particularly for those used in automotive tires, is carbon black. However, silica has been also recognized as notable filler for high performance tires with good wet grip and low rolling resistance and it is widely used as reinforcing filler for tire tread [7]. Silica particles have become more important in tire applications since the introduction of the Green Tire ${ }^{\circledR}$ by Michelin in 1992. As an alternative to carbon black filler, silica is typically incorporated in low rolling resistance tires in their tread compounds providing reduction in low-frequency hysteresis without compromising traction. As a filler, silica has greater reinforcing power, such as improving tear strength, abrasion resistance, age resistance and adhesion properties than carbon black [8]. Silica filled rubber compounds show low hysteresis losses in comparison with carbon black filled rubber compounds. Nevertheless, due to the strong inter-particle hydrogen bonds between the hydroxyl groups, silica particles are highly inclined to agglomeration. A widely held view is that formation of filler inter-aggregate associations and their networking are responsible for the high degree of reinforcement [9]. At the same time, the related effects - deagglomeration and breakdown of the filler network structure, are responsible

\footnotetext{
* Corresponding author. Tel.: +371-67543303; fax: +371-67820467

E-mail address: olesja.starkova@pmi.lu.lv(O. Starkova)
}

for the specific features of filled rubbers, such as stress softening or Mullins effect, and hysteresis [1, 2].

The Mullins effect is a particular feature of the stressstrain behavior of rubber, particularly rubber compounds containing fillers. It is named after L. Mullins, who made extensive studies of the effect [10]. The effect is also known as the softening phenomenon and is referred to as stress-softening, stretch-induced softening, and/or cyclic softening. The effect is mainly associated with a significant reduction in the stress at a given level of strain at unloading as compared with the stress at initial loading on the first and subsequent cycles in stress-strain cyclic tests of fixed amplitude. The effect of similar nature - the Payne effect - is observed in dynamic tests under cyclic loading conditions with small strain amplitudes and referred to a dependence of the viscoelastic storage modulus on the amplitude of the applied strain [3,11]. Although Mullins identified softening to occur in both unfilled and filled elastomers, its effect is far more pronounced in filled elastomers and therefore is frequently identified to be a filled elastomer phenomenon.

Most of elastomer softening theories is based on two concepts. According to the first theory, rubber-filler attachments are formed by incorporation of fillers into the crosslinked rubber network. These attachments provide additional restrictions on deformation of rubber network that appears in the increased stiffness $[1,9]$. However, their breakdown or weakening results in softening of filled rubbers. The second theory originates from Mullins himself, who considered stretch-induced softening to be a result of evolution of the microstructure due to a quasiirreversible rearrangement of rubber molecular networks only, which involves breaking and reforming of crosslinks during extension, residual load orientation of network chains persisting after recovery and breaking of network 
chains $[10,12]$. There is also the third widely held view that softening is related to a specific interaction in the filler inter-aggregate associations. Taking into account nanoscopic size of the filler for which it is impossible to neglect either filler-filler interaction or the filler-matrix interaction, all three mechanisms are mostly responsible for development of the softening phenomenon. Contribution from the filler structure, interphase between the rubber and particles, and from the rubber matrix to the mechanisms of softening varies noticeably depending on a material and test conditions [13, 14].

Evaluation of the hysteresis losses is a task of great importance especially for rubbers used for tires in automotive industry. Hysteresis is an extent of energy dissipated as heat due to cyclic deformation and is also considered as a quantitative characteristic of softening. Strain dependences of the hysteresis losses can provide useful information on the mechanisms of softening and their contribution on different stages of extension $[5,15]$.

Despite on a large variety of papers devoted to investigation of the softening phenomenon of various elastomers, there is still a lack of data on systematic experimental investigation of the effect of rigid inclusions on softening and quantitative estimation of this effect for rubbers with various filler contents. The aim of the present study was to estimate Mullins effect in silica filled styrenebutadiene rubber and to determine softening characteristics and their dependencies on the filler volume content; to reveal the mechanisms of softening on different stages of deformation by investigating strain dependencies of the hysteresis losses of the neat and filled rubber.

\section{MATERIALS AND METHODS}

The material under investigation is styrene-butadiene rubber, neat and filled with $\Phi=9,14$ and 21 vol. \% of silica particles. Dumbbell samples were cut from about 2 mm thick plates according to the standard DIN-ISO-527-2 $5 \mathrm{~A}$.

Uniaxial tensile tests up to failure and uniaxial cyclic tests were performed on a Zwick 2.5 universal testing machine by using $2.5 \mathrm{kN}$ load cell at a constant crosshead speed $50 \mathrm{~mm} / \mathrm{min}$, which corresponded to the nominal strain rate $0.033 \mathrm{~s}^{-1}$. Samples were preloaded up to $0.5 \mathrm{~N}$. In cyclic tests, five loading-unloading cycles were applied to each sample up to the strain corresponded to 0.25 of the strain at failure according to the scheme shown in Fig. 1. One-minute time interval was held between the cycles, necessary for reconfiguration for the following measurements. At least three duplicate samples with the same filler content were tested. The tests were performed under room conditions under an average $T=22{ }^{\circ} \mathrm{C}$.

The displacement, which is proportional to the nominal strain, was controlled by displacement of white markers marked on the parallel part of each sample. Two marks in axial and two marks in lateral direction of samples were drawn in order to control longitudinal and transversal strains, respectively. Images of the operative zone of samples were made by a high resolution photo camera at fixed time intervals. Displacements were evaluated by handling photo images by using a special software [16].

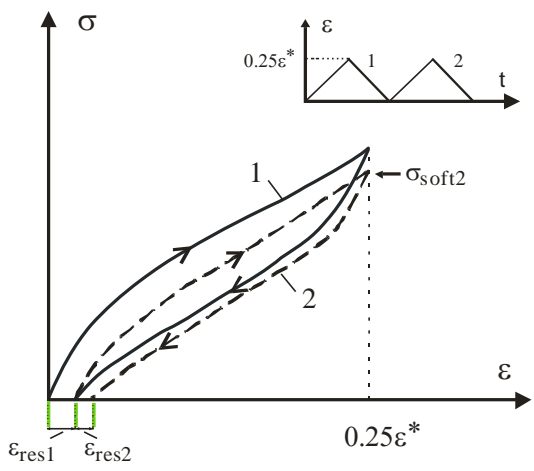

Fig. 1. Scheme of determination of softening characteristics and the test regime (inset)

The residual strain $\varepsilon_{\text {res }}$ was calculated as a difference between the strain values at the start of loading and the end of unloading. The softening stress $\sigma_{\text {soft }}$ is the stress value achieved at the end of loading path (Fig. 1). The hysteresis losses were calculated by the ratio of areas under loading and unloading curves, i.e.

$H=\frac{S_{\text {load }}-S_{\text {unload }}}{S_{\text {load }}}=1-\frac{S_{\text {unload }}}{S_{\text {load }}}$,

where $H$ is hysteresis losses; $S_{\text {load }}$ and $S_{\text {unload }}$ are areas under loading and unloading curves, respectively. Strain dependences of the hysteresis losses were determined by evaluation of the corresponding areas under loading and unloading curves at current strain values, while the total losses were calculated for the whole loop of each cycle.

Relative volume changes were calculated by formula

$\frac{\Delta V}{V_{0}}=\left(1+\varepsilon_{1}\right)\left(1+\varepsilon_{2}\right)^{2}-1$,

where $V_{0}$ is volume of a sample in undeformed state; $\varepsilon_{1}$ and $\varepsilon_{2}$ are longitudinal and transversal strains, respectively. It was assumed that strain field in two normal to loading axis directions is homogeneous, i.e. $\varepsilon_{2}=\varepsilon_{3}$ [17]. Since all strain dependences in this paper are given for longitudinal strains, the designation $\varepsilon_{1}=\varepsilon$ has been introduced. The results are given in nominal strain and nominal stress units.

\section{RESULTS AND DISCUSSION}

Stress-strain diagrams up to failure of the neat and filled rubber samples are shown in Fig. 2. The elastic and strength characteristics of the neat rubber were considerably improved by incorporating silica particles.

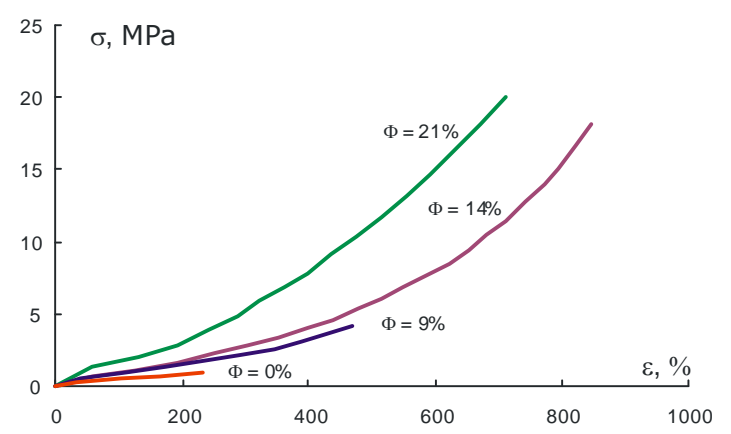

Fig. 2. Typical stress-strain curves up to failure

The higher the filler content, the higher the reinforcement of the rubber. For example, the strength and strain at failure of the neat rubber and rubber filled with 
21 vol.\% of silica differs for about 20 and 3 times, respectively. Significant increase of the slope of stressstrain curves with an increase of the filler volume content is observed that indicates to an increase of the elastic modulus and general reinforcement of the filled material. Deformational characteristics of rubber compounds are given in Table 1.

Table 1. Deformational characteristics of rubber compounds

\begin{tabular}{|c|c|c|c|}
\hline $\begin{array}{c}\text { Filler } \\
\text { content } \\
\Phi, \%\end{array}$ & $\begin{array}{c}\text { Strength } \\
\sigma^{*}, \mathrm{MPa}\end{array}$ & $\begin{array}{c}\text { Strain at } \\
\text { failure } \\
\varepsilon^{*}, \%\end{array}$ & $\begin{array}{c}\text { Elastic } \\
\text { modulus } \\
E, \text { MPa }\end{array}$ \\
\hline 0 & $1.0( \pm 0.05)$ & $252( \pm 16)$ & $0.55( \pm 0.02)$ \\
\hline 9 & $4.6( \pm 0.5)$ & $514( \pm 37)$ & $1.22( \pm 0.07)$ \\
\hline 14 & $18.0( \pm 1.6)$ & $866( \pm 45)$ & $2.02( \pm 0.2)$ \\
\hline 21 & $21.4( \pm 1.4)$ & $711( \pm 33)$ & $7.58( \pm 0.74)$ \\
\hline
\end{tabular}

Typical stress-strain curves of the rubber for the first loading-unloading cycle are given in Fig. 3 a. Besides the reinforcement effect, deformation behavior of the filled rubber is characterized by its softening, which appears in hysteresis and residual strains. Under subsequent loading, stress-strain curves have similar trends for all filled samples. Typical loading-unloading curves are shown in Fig. $3 \mathrm{~b}$ for the rubber filled with 21 vol. $\%$ of silica as an example.
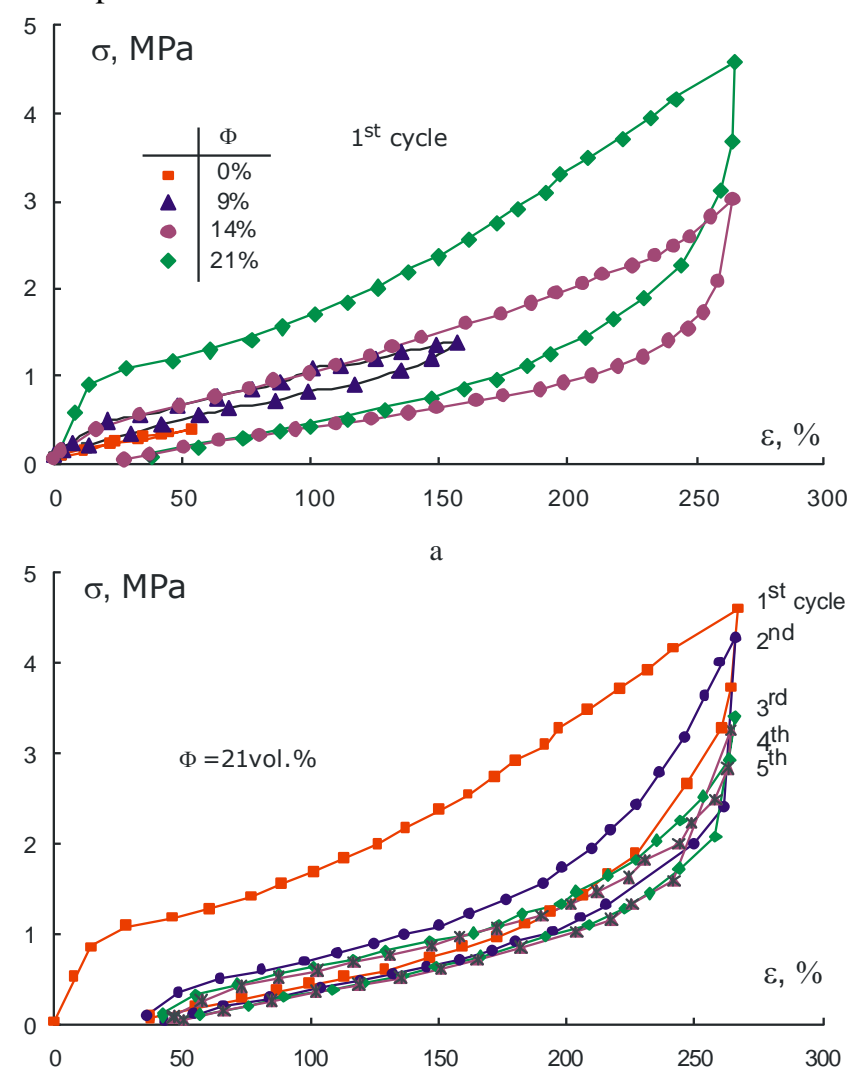

b

Fig. 3. a-stress-strain behavior in the first loading-unloading cycle for different rubber compounds; $b$-in five loadingunloading cycles for the compound with $\Phi=21 \%$

The loading curves are similar for the first three cycles, but the stress value reached in repeated cycles is smaller than that in the first cycle. The curves for the fourth and fifth cycles practically fit to each other that indicate to stabilization of the sample state and completion of the softening processes. Softening characteristics of the neat and filled rubber are given in Table 2. The residual strain and softening stress significantly increase with an increase of the filler volume content and decrease in subsequent cycles. The largest change in softening characteristics is observed between the first and second loading cycles. Interesting to note that even under significant softening in the first two cycles, the unloading curves are practically independent of the number of cycle, i.e. stress-strain curves at unloading are independent of loading history of the material.

The hysteresis losses are another characteristic of softening. As seen from Fig. 3 a, the hysteresis loops are the greater, the higher the filler content of the sample, while energy losses due to deformation of the neat rubber are negligible compared to these of the filled rubber compounds. Area of the hysteresis loops, i.e. the total losses, gradually decreased with subsequent loadingunloading cycles (Fig. $3 \mathrm{~b}$ ) and remained practically unchanged after the fourth cycle. Fig. 4 shows that the overall tendency between the rubber compounds remains the same: the higher the filler content, the greater the hysteresis losses.

Table 2. Softening characteristics of rubber compounds

\begin{tabular}{|c|c|c|c|c|c|c|c|c|}
\hline \multirow{3}{*}{$\begin{array}{c}\text { Cycle } \\
\text { No. }\end{array}$} & \multicolumn{4}{|c|}{$\begin{array}{c}\text { Residual strain } \\
\varepsilon_{\text {res }}, \%\end{array}$} & \multicolumn{5}{c|}{$\begin{array}{c}\text { Softening stress } \\
\sigma_{\text {soft }}, \mathrm{MPa}\end{array}$} \\
\cline { 2 - 10 } & \multicolumn{9}{|c|}{$\Phi, \%$} \\
\hline & 0 & 9 & 14 & 21 & 0 & 9 & 14 & 21 \\
\hline 1 & 0 & 3.7 & 27.5 & 36.8 & $(0.4)$ & $(1.4)$ & $(2.9)$ & $(4.6)$ \\
\hline 2 & 0 & 2.6 & 6.2 & 6.6 & 0.38 & 1.37 & 2.2 & 4.2 \\
\hline 3 & 0 & 2.2 & 4.4 & 5.2 & 0.38 & 1.32 & 1.8 & 3.1 \\
\hline 4 & 0 & 1 & 2.6 & 4.5 & 0.38 & 1.27 & 1.7 & 3.0 \\
\hline 5 & 0 & 1 & 2.6 & 4.5 & 0.38 & 1.27 & 1.7 & 3.0 \\
\hline
\end{tabular}

The hysteresis losses are a measure of energy dissipated under cyclic deformation of rubber. Energy is partly stored elastically and partly dissipated as heat due to molecular friction. Total consumption of energy, i.e. work done on deformation of a sample, corresponds to an area under the stress-strain curve [15]:

$W=\int_{0}^{\varepsilon} \sigma d \varepsilon$

For interpretation of the experimental data from physical point of view, Eq. 1 can be written as

$H=\frac{W-W_{\text {stor }}}{W}=\frac{W_{\text {dis }}}{W}$,

where $W, W_{\text {stor }}$, and $W_{\text {dis }}$ are the total, stored an dissipated energies, respectively. Since the hysteresis losses are determined as a ratio of the dissipated energy to the total energy of deformation, strain dependences of the hysteresis losses can be used as a tool for evaluation of the contribution of each energy component to the total energy at different stages of deformation.

Strain dependences of the hysteresis losses in the first loading-unloading cycle are shown in Fig. 5 a. For the filled rubber, hysteresis losses are monotonically decreasing function on strain. Despite on growing absolute values of the losses with an increase of silica content, the rate of changes of the losses (slope of the curves) is significantly lower for the rubber with high filler content. Strain dependences of the hysteresis losses in five loadingunloading cycles for rubber samples filled with 21 vol.\% 
of silica are shown in Fig. 5 b as an example. The greatest difference in the absolute values and slope of the curves is observed for the first and second cycles, while the curves of the fourth and fifth cycles practically fit to each other.

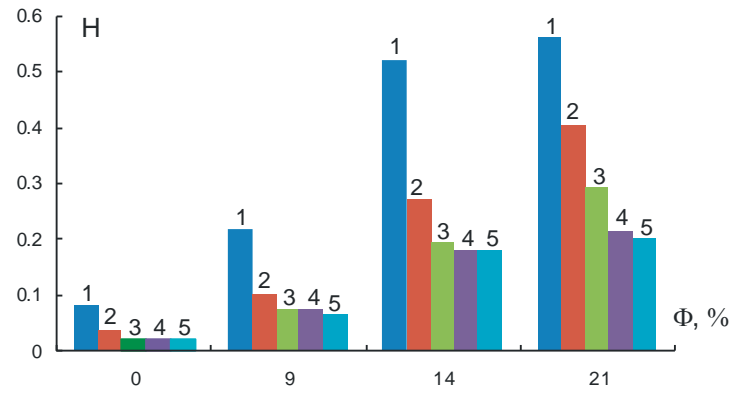

Fig. 4. Total hysteresis losses of rubber compounds in five loading-unloading cycles

From the point of view of molecular structure, rubber is an assemblage of chaotically contracted entangled flexible molecular chains, which conformations are random and mostly reversible. Since molecular chains are crosslinked by chemical or mechanical junctions, rubber is a spatial interpenetrating macroscopic network and its deformation is a result of stretching of contracted molecular chains to energetically unfavorable extended conformations. After load removal, chains between the crosslinks rapidly recover to their initial state due to Brownian motion, thus contracting junctions to the equilibrium state. Incorporation of filler particles into rubbers, changes their molecular structure by expanding the number and type of the bonds. In addition to chemical crosslinks and local secondary intermolecular bonds, bonds between the filler and elastomer appear in filled rubbers, which are mainly adsorptive-type bonds [9]. The elastomer-particle bonds intermediate between the strength of chemical and intermolecular bonds between the chains of rubber that mostly determines ability of filled elastomers to restore their internal structure $[6,15]$.

In filler-reinforced rubbers, energy losses during deformation are caused by two main reasons. The first reason is related to the internal viscosity of the rubber caused by motion and rearrangement of macromolecular chains and sliding of their segments relative to each other. The second reason for the energy dissipation is destruction of the structure of filled elastomers, which includes breaking and redistribution of bonds in the network formed by the filler particles and the bonds formed by the particles with adjacent molecules [15]. Contribution of two damage mechanisms to the total energy losses is different on different stages of extension: an intensive destruction of the inter-aggregate associations of the filler particles appears under small strains, while the particle-elastomer bonds are broken under large elongations. In addition, significant energy losses with growing strain are caused by irreversible processes in the rubbery phase associated with a nonuniform displacement of network junctions due to complete extension of short chains and sliding of rubber chains relative to each other [5].

In unfilled elastomers, the main contribution to the energy losses comes from the internal viscosity and segmental mobility of the material that hampers rearrangement of molecular chains of the crosslinked network. At unloading, the molecular chains practically restore to their initial state, although some rearrangement of the structure still takes place that appears in slight hysteresis losses (Fig. 3). After subsequent loading, rearrangement of the rubber structure caused by deformation is completely recoverable and, as experimental data showed, the hysteresis is negligible after the second loading-unloading cycle (Fig. 4). It is noteworthy to point out nonmonotonic character of the strain dependence of the hysteresis losses of the neat rubber shown in Fig. 5 a.
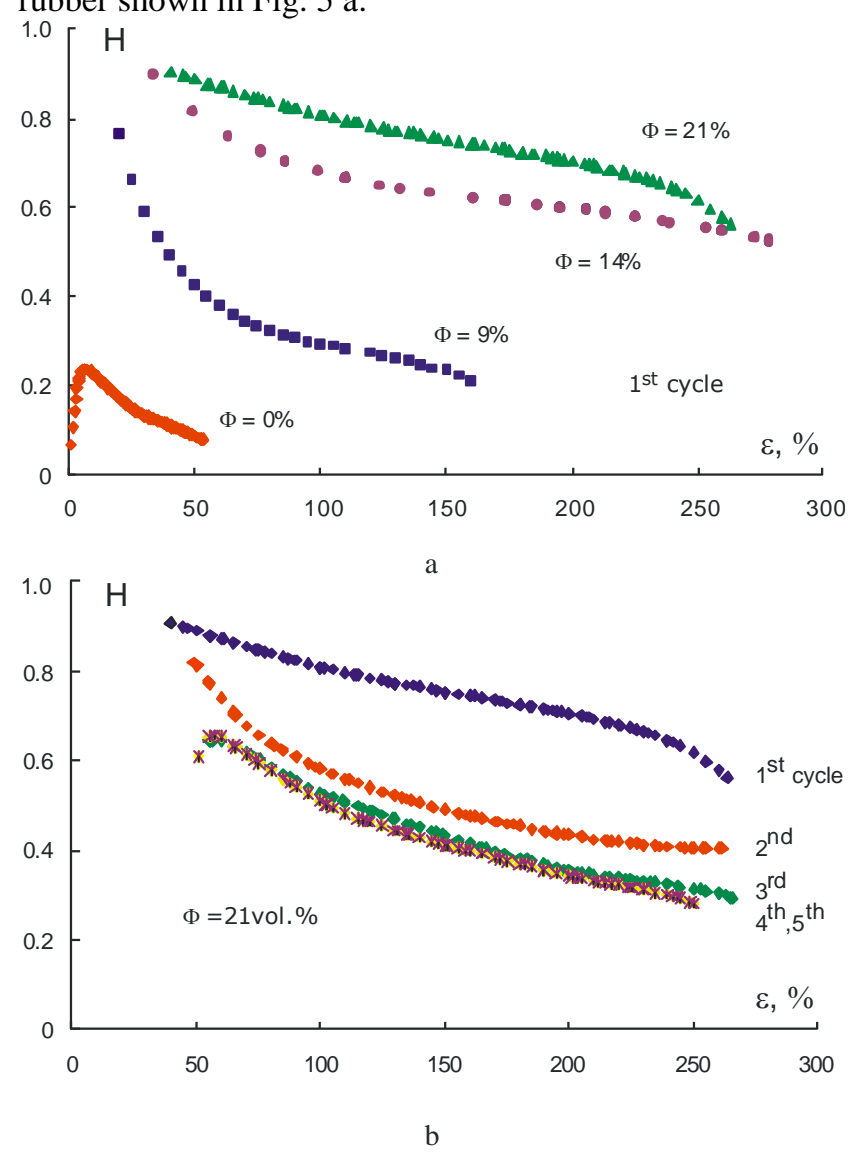

Fig. 5. a-strain dependences of the hysteresis losses in the first; $\mathrm{b}$-in five loading-unloading cycles

Occurrence of the maximum on the curve is caused by variation of the ratio between the energy losses and energy of deformation. Under small strains, straightening of nonmoving segments of the elastomeric network takes place. This process is characterized by insignificant both, dissipated and stored, energies. However, contribution of the energy losses to the total energy of deformation is higher at the initial stage of deformation than that from the elastically stored energy, thus maximum on the curve appears. Segmental mobility of the rubber increases with growing strain and contribution of the dissipated energy increases. At the same time, contribution of the stored energy into the total energy of deformation also noticeably increases under elastic deformation of segments of molecular chains. Decrease of the hysteresis losses of the neat rubber in Fig. 5 a assumes that contribution of the stored energy is greater than that of the dissipated energy when strains are higher than $10 \%$.

For filled rubbers, as Fig. 5 a shows, the strain dependences of the hysteresis losses are generally similar 
to those of the neat rubber. The more intensive breakage of the filler inter-aggregate associations occurs under small deformations accompanied by a sharp increase of the hysteresis losses with an increase of silica content, whereupon the main contribution comes from the rubbery phase and the losses decrease $[1,5]$. However, there are some features related with the specific interactions between the filler and rubber matrix. Silica particles form the network structure, which consists of chains of different lengths. During extension, different structural elements are involved and the structure does not fail wholly at once. The decrease of the losses with growing strain is less pronounced for the filled rubber than for the neat one. Since the hysteresis losses are determined by the ratio of the dissipated energy and the strain energy, the lower rate of the losses' decrease indicates to the higher contribution of the dissipative energy than from the strain energy. The greater the filler content, the higher this contribution.
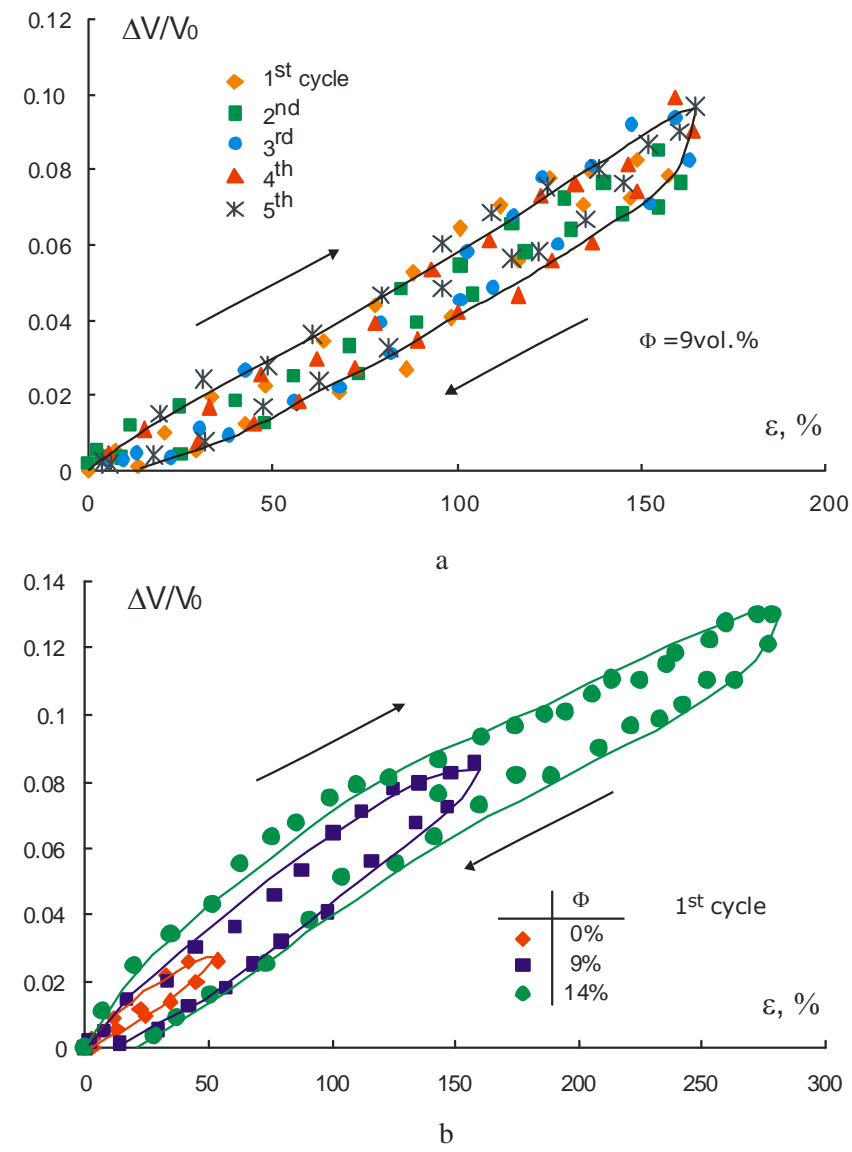

Fig. 6. a-relative volume changes as function of strain in five loading-unloading cycles for the compound $\Phi=9 \%$; in the first loading-unloading cycle for different rubber compounds

The intensity of breakage of the silica associations and silica-rubber bonds is the greatest during the first loading cycle. The former is related to a reduction of the amount of losses at the beginning of deformation, while the latter appears as a decrease of the slope of the strain dependences of the hysteresis losses with the growing number of cycles (Fig. 6 a). Moreover, both breakage mechanisms approach a steady state after four loadingunloading cycles and the hysteresis losses at subsequent cycles repeat each other. It is interesting to note that the maximum on the starting segment of the losses-strain curve appears henceforth, similarly as for the neat rubber. This is caused by a removal of the residual strain on the stress-strain curves that, in turn, indicates to reversibility of the rearrangement processes in the material's structure. Besides, slope of the strain dependences of the hysteresis losses after the first several cycles was similar for all compounds. This all proves a fact that once the softening effect caused by the specific silica and silica-rubber interactions is removed the deformation behavior is mainly determined by the rubbery phase.

An ability of the neat and filled rubber to structural recovery can be also demonstrated by volume changes of the materials. Strain dependences of volume changes for the rubber filled with 9 vol.\% of silica shown in Fig. 6 a demonstrate that volume changes are also characterized by hysteresis, however the curves for various cycles practically fit to each other. Volume changes tend to zero when load is completely removed, i.e. they are reversible. Similar trends were observed for all rubber compounds. Volume changes of the neat and filled rubber in the first cycle of deformation are compared in Fig. 6 b. As expected, volume changes at strain values up to 0.25 of the strain at failure increase with the amount of filler and reach values up to $10 \%$ and higher. Nevertheless, in the considered strain range, volume changes are completely reversible independently from the filler volume content. Comparing the data at the same strain level, for example at $\varepsilon=50 \%$, one can notice relatively small difference in the absolute values of volume changes for the neat and filled rubber. As it was mentioned above, deformation of rubber is mainly caused by stretching of the folded intermolecular chains, which owing to crosslinks form the interpenetrating network. Under deformation, an increase of inter-chain (not of inter-molecular spaces) takes place in this structure, i.e. rearrangements of these chains are reversible and occur without volume changes [15]. In filled rubbers, filler particles contribute to the formation of additional crosslinks, which results in material ability to exhibit large strains [13]. Nevertheless, deformation is mainly caused by conformational changes of the elastomer matrix and, as a result, volume changes are weakly dependent on the filler volume content and number of cycles. Similar conclusions were also made in [17-20].

\section{CONCLUSIONS}

The following features of softening behaviour of the silica filled styrene-butadiene rubber are pointed out: i) the pre-stretched material is much more compliant than the initial one, ii) the Mullins effect is the most noticeable in the first and second cycle of loading, iii) subsequent loading causes further stress softening but the degree of damage of the material structure is considerably lower than that after the first cycle, iv) the residual strains appear mostly after the first cycle and after repeated cycles their values remain practically unchanged, and v) the degree of softening increases with increasing volume content of the filler, while softening of the unfilled rubber is insignificant.

The hysteresis losses are the greater, the higher the filler content is, while they are almost negligible for the neat rubber. The total losses are the greatest during the first 
cycle, gradually decrease with subsequent cycles and stabilize after several loading cycles. The energy losses due to deformation are caused by two main reasons: internal viscosity of the rubber and damage or rearrangement of the microstructure of the elastomer matrix and the filler-formed network. Contribution of different damage mechanisms to the total energy of deformation was estimated by strain dependences of the hysteresis losses at different loading cycles for the neat and filled rubber. At the very beginning of deformation, energy losses of the filled rubber are associated with intensive destruction of the filler network, while under large elongations the main contribution to the energy dissipation comes from the rubbery phase and from breakage of the bonds between the rubber chains and the fillers. After repeated loading-unloading cycles, the filler-filler and filler-elastomer bonds become weaker and come to a steady state. Then, the material structure is practically recovered in each cycle and hysteresis losses are caused by changes in the rubbery phase only. Ability of the neat and filled rubber to almost completely restore their structure is confirmed by recoverable volume changes.

\section{Acknowledgments}

The ESF project No. 2013/0017/1DP/1.1.1.2.0/13/ APIA/VIAA/063 is acknowledged for the financial support of this work.

\section{REFERENCES}

1. Qi, H. J., Boyce, M. C. Stress-strain Behavior of Thermoplastic Polyurethanes Mechanics of Materials 37 2005: pp. 817-839.

2. Cheng, M., Chen, W. Experimental Investigation of the Stress-stretch Behavior of EPDM Rubber with Loading Rate Effects International Journal of Solids and Structures 40 2003: pp. $4749-4768$.

3. Frohlich, J., Niedermeier, W., Luginsland, H. D. The Effect of Filler-filler and Filler-elastomer Interaction on Rubber Reinforcement Composites A 36 2005: pp. 449-460.

4. Gauthier, C., Reynaud, E., Vassoille, R., LadouceStelandre, L. Analysis of Non-linear Behavior of Silica Filled Styrene-butadiene Rubber Polymer 45 2004: pp. $2761-2771$.

5. Kucherskii, A. M. Hysteresis Losses in Carbon-black-filled Rubbers under Small and Large Elongations Polymer Testing 24 2005: pp. 733-738.
6. Reznikovsky, M., Lukomskaya, A. I. Mechanical Testing of Caoutchouc and Rubbers. Chemistry, Moscow, 1968.

7. Takaaki, M. Recent Technological Trends of Styrenebutadiene Rubber Journal of the Society of Rubber Industry 78 (2) 2005: pp. 46-50.

8. Peng, C., Gopfert, A., Drechsler, M., Abetz, V. Smart Silica-Rubber Nanocomposites in Virtue of Hydrogen Bonding Interaction Polymer Advanced Technology 16 2005: pp. $770-782$.

9. Morozov, I. A., Svistkov, A. L., Heinrich, G., Lauke, B. Macromolecular Compounds (in Russian) 3 (49) 2007: pp. 456-464.

10. Mullins, L., Tobin, N. R. Theoretical Model for the Elastic Behavior of Filler-reinforced Vulcanized Rubber Rubber Chemical Technology 30 1957: pp. 555-571.

11. Payne, A. R. The Dynamic Properties of Carbon-blackloaded Natural Rubber Vulcanizates Journal of Applied Polymer Science 6(19) 1962: pp. 57-63.

12. Göktepe, S., Miehe, C. A Micro-macro Approach to Rubber-like Materials. Part III: The Micro-sphere model of Anisotropic Mullins-type Damage Journal of Mechanics and Physics of Solids 53 2005: pp. 2259-2283.

13. Aniskevich, K., Starkova, O., Aniskevich, A. Viscoelastic Properties of Silica Filled Styrene-butadiene Rubber under Uniaxial Tension Mechanics of Composite Materials 46 2010: pp. $375-386$. http://dx.doi.org/10.1007/s11029-010-9154-x

14. Aniskevich, K., $\quad$ Starkova, O., Aniskevich, A. Deformational Properties of Filled Rubber under Quasistatic Loading Journal of Applied Polymer Science 123 (3) 2012: pp. $1621-1629$. http://dx.doi.org/10.1002/app.34557

15. Liebowitz, H. Fracture. Vol. 7. Fracture of Nonmetals and Composites. New York, 1972.

16. Aniskevich, A., Kalnroze, Z. Materials Technology Instruments (in Russian) 13 (1) 2008: pp. 105-110.

17. Starkova, O., Aniskevich, A. Poisson's Ratio and the Incompressibility Relation for Various Strain Measures with the Example of a Silica-filled SBR Rubber in Uniaxial Tension Tests Polymer Testing 29 2010: pp. 310-318.

18. Le Cam, J.-B. A Review of Volume Changes in Rubbers: the Effect of Stretching Rubber Chemistry and Technology 83 (3) 2010: pp. 247-269.

19. Le Cam, J.-B., Toussaint, E. Cyclic Volume Changes in Rubber Mechanics of Materials 41 2009: pp. 898-901.

20. Kazina, E., Starkova, O., Aniskevich, A. Volume Changes in Filled Rubber under Uniaxial Cyclic Loading Materials Science (Medžiagotyra) 17 (3) 2011: pp. 271-275. 